\title{
Determinação de Parâmetros Hemodinâmicos Através do Ecocardiograma Bidimensional com Doppler: Ferramenta para Busca de Otimização Terapêutica em Pacientes Ambulatoriais com Insuficiência Cardíaca Congestiva
}

\author{
Determination of Hemodynamic Parameters Using Doppler Two-dimensional \\ Echocardiography: A Searching Tool for Therapeutic Optimization in Patients \\ with Congestive Heart Failure on an Outpatient Care Follow-up
}

\author{
Dora V. Palombini, Luis E. Rohde, Leticia Crestana, Lívia Goldraich, Marta Pereira Lima, \\ Candice Campos, Nadine Clausell \\ Porto Alegre, RS
}

Um dos principais problemas no manejo dos pacientes com insuficiência cardíaca grave são as freqüentes hospitalizações por descompensação, que, além de causar grande ônus para o sistema de saúde previdenciário, é uma das principais causas da perda de qualidade de vida destes pacientes. Em média 30 a 50\% dos pacientes reinternam após 3 a 6 meses da alta hospitalar ${ }^{1}$. Uma metanálise recente indicou que a freqüência dessas rehospitalizações pode ser reduzida através de acompanhamento constante de uma equipe multidisciplinar de médicos e enfermeiras em clínica especializada em insuficiência cardíaca ${ }^{2,3}$. Portanto, um acompanhamento meticuloso com tratamento individualizado e dirigido, para situação específica e atual de cada paciente, poderia trazer benefícios a médio e longo prazo no tratamento ambulatorial dos pacientes crônicos com insuficiência cardíaca congestiva moderada a grave. Neste artigo apontamos as potenciais aplicações de métodos como o ecocardiograma para o manejo otimizado de pacientes com insuficiência cardíaca congestiva e apresentamos resultados iniciais da utilização deste instrumento para guiar sua terapêutica.

Peculiaridades no desenvolvimento e nas manifestações de congestão e baixo débito na insuficiência cardíaca congestiva Em pacientes com insuficiência cardíaca congestiva uma importante causa de rehospitalização e/ou visita à sala de emergência está associada a quadros congestivos ${ }^{4,5}$. Por outro lado, o diagnóstico clínico de estados congestivos pode ser, particularmente, difícil em pacientes crônicos, devido a uma série de adaptações fisiopatológicas que fazem com que as manifestações clínicas de congestão sejam sutis nesses pacientes, freqüentemente levando à sub-utilização de diuréticos e vasodilatadores ${ }^{6}$, o que, por sua vez, contribui para risco de descompensação.

Hospital de Clínicas de Porto Alegre e Programa de Pós-Graduação em Ciências Cardiovasculares: Cardiologia

Endereço para correspondência: Dra. Nadine Clausell - Rua Honório Silveira Dias, 873/901 - Cep 90550-150 - Porto Alegre - RS

E-mail: nclausell@cardiol.br / clausell@portoweb.com.br

Enviado em 30/03/2004 - Aceito em 15/07/2004
A identificação de pressões de enchimento ventricular elevadas na insuficiência cardíaca congestiva não segue o mesmo padrão dos achados clínicos semióticos encontrados nos quadros agudos, a ser considerada para tomada de decisões terapêuticas. Um dos principais sinais e sintomas de congestão é a elevação da pressão venosa jugular e a ortopnéia. A avaliação clínica da pressão venosa jugular requer técnica cuidadosa, pois parece ser o sinal clínico mais importante na avaliação do real estado congestivo dos portadores de insuficiência cardíaca congestiva ${ }^{7}$.

Sabe-se que na insuficiência cardíaca congestiva, a concordância entre estimativas de pressões à direita e à esquerda é de $80 \%$, pois Drazner e cols. ${ }^{8}$ demonstraram que através de uma pressão atrial direita $(P A D)>$ ou $<10 \mathrm{mmHg}$ é possível estimar uma pressão atrial esquerda (PAE) $>$ ou $<22 \mathrm{mmhg}$, respectivamente $\mathrm{e}^{8,9}$. Outros fatores, como: compressão abdominal com refluxo hepato-jugular positivo, resposta pressórica tipo "raiz quadrada" à manobra de Valsalva e hiperfonese de $2^{\circ}$ bulha também podem sinalizar elevação das pressões em cavidades direitas que, por sua vez, podem estimar pressões altas à esquerda ${ }^{9,10}$.

Devido à drenagem linfática compensatória existente cronicamente em portadores de insuficiência cardíaca congestiva, estertores pulmonares estão ausentes em mais de $80 \%$ dos casos, mesmo existindo pressões de enchimento elevadas. Já os sintomas de dispnéia e cansaço se devem ao fato de que mesmo que o fluido intersticial residual não comprometa a oxigenação, pode provocar uma restrição na inspiração e redução da complacência pulmonar ${ }^{6}$.

Edema de membros inferiores ocorre em apenas 25\% dos pacientes com insuficiência cardíaca congestiva abaixo dos 70 anos de idade, enquanto que nos mais velhos pode ser causado mais por fatores locais do que por elevação da pressão venosa central propriamente dita. Com estes dados é possível sugerir que edema periférico e pressão venosa jugular elevada sejam mais específicos do que sensíveis para detectar pressões de enchimento esquerdas elevadas.

A $3^{\circ}$ bulha está presente na maioria dos pacientes em insufi- 
Determinação de Parâmetros Hemodinâmicos Através do Ecocardiograma Bidimensional com Doppler: Ferramenta para Busca de Otimização Terapêutica em Pacientes Ambulatoriais com Insuficiência Cardíaca Congestiva

ciência cardíaca congestiva, e, em alguns pacientes, pelo contrário, nunca é identificada. Cumpre ressaltar que o grau de concordância, mesmo por médicos experientes, é moderado ou baixo sobre a presença ou não de $3^{\circ}$ bulha. Esse fato provavelmente está ligado à perda de treinamento auscultatório em tempos de métodos diagnósticos mais sofisticados e de fácil acessibilidade ${ }^{11-13}$. Alguns autores sugerem que a $3^{\circ}$ bulha não se constitui em um parâmetro de avaliação confiável do estado congestivo dos pacientes com insuficiência cardíaca congestiva ${ }^{6}$. Ainda assim, a presença de $3^{\circ}$ bulha e a elevação da pressão venosa jugular na insuficiência cardíaca congestiva estão associados, independentemente, com maior incidência de hospitalizações por insuficiência cardíaca $(R R=1,32, p<0,01)$, morte ou hospitalizações por insuficiência cardíaca $(R R=1,30, p<0,005)$ e morte por insuficiência cardíaca $(R R=1,37, p<0,05)^{4}$.

A razão pela qual a elevação da pressão venosa jugular ou presença de $3^{\circ}$ bulha está associada a um risco elevado de progressão da insuficiência cardíaca congestiva não é clara. Pressão venosa jugular elevada reflete aumento da pressão atrial direita, que, por si só, se correlaciona com pressões esquerdas elevadas nos pacientes com insuficiência cardíaca congestiva. Estas, por sua vez, estão associadas com mau prognóstico, possivelmente, pela apoptose desencadeada pela tensão parietal miocárdica e pela ativação do sistema nervoso simpático ${ }^{14,15}$. A $3^{\circ}$ bulha, mais ligada à baixa complacência do ventrículo esquerdo e a pressões e velocidades de enchimento elevadas, também tem sido associada a um mau prognóstico por distúrbio na diástole em pacientes com disfunção sistólica ${ }^{4}$.

Um dos poucos instrumentos clínicos úteis à beira do leito na avaliação dos pacientes com insuficiência cardíaca congestiva é a pressão de pulso (pressão sistólica - pressão diastólica / pressão sistólica), que, quando < que $25 \%$ estima um índice cardíaco de 2,2 L/min/m $/ \mathrm{m}^{2-6}$. Também se pode utilizar com uma acurácia de $93 \%$, a razão entre a pressão de pulso obtida com a respiração normal e a pressão de pulso durante a manobra de Valsalva, para estimar a pressão atrial esquerda ${ }^{16}$.

Além destas características, em pacientes com insuficiência cardíaca congestiva e diâmetros ventriculares aumentados, o grau de regurgitação mitral é, via de regra, significativo, contribuindo de forma importante para o aparecimento de sinais e sintomas de congestão e baixo débito. 0 mecanismo responsável pela regurgitação mitral na insuficiência cardíaca congestiva é a não coaptação adequada dos folhetos mitrais devido à dilatação do anel mitral e comprometimento da geometria do aparato subvalvular ${ }^{17}$. Com a compensação do quadro congestivo e redução do volume ventricular e atrial esquerdo, a insuficiência mitral e o orifício regurgitante efetivo reduzem-se consideravelmente. Com isto, o "roubo" no volume sistólico de mais de 50\%, que ocorre nos pacientes sintomáticos em repouso, é redistribuído, e há um aumento do volume sistólico efetivo ventricular esquerdo ${ }^{18}$. Devido ao fato dos sintomas dominantes serem secundários à congestão, o conseqüente alívio dos sintomas em repouso se deve principalmente à redução de pressões de enchimento elevadas. Outros fatores afetados positivamente pela redução das pressões de enchimento são: a melhora da perfusão coronariana, a redução da ativação neurohormonal com redução da liberação de norepinefrina e do estresse parietal ${ }^{14,19,20}$, melhora da capacidade funcional ${ }^{21}$, redu- tão hepática e mais adiante da desnutrição progressiva dos pacientes crônicos ${ }^{22}$.

Ao contrário daqueles pacientes no pós-infarto agudo do miocárdio, em que o miocárdio enrijecido, ainda não dilatado, se beneficia de pressões de enchimento altas, pacientes com ventrículos cronicamente dilatados possuem sarcômeros maximamente alongados, em que uma maior sobrecarga de pressão ou volume não irá alongá-lo ainda mais, mas sim, aumentar a tensão da parede e a incompetência valvular. Portanto, a redução das pressões de enchimento para níveis normais ou baixos com diuréticos e vasodilatadores, se reflete em aumento do volume sistólico. Este aumento se deve não a uma melhora na fração de ejeção, que pouco se eleva, mas a grande redução do volume sistólico perdido na regurgitação mitral ${ }^{23}$.

Otimização hemodinâmica na insuficiência cardíaca - Os objetivos básicos que regem a terapia clínica da insuficiência cardíaca guiada por otimização hemodinâmica são aqueles definidos por Stevenson e cols.; especificamente - pressão atrial esquerda de $15 \mathrm{mmHg}$ ou menos, pressão de átrio direito $\leq \mathrm{a} 8 \mathrm{mmHg}$, resistência vascular sistêmica em torno de 1.200 dinas $/ \mathrm{seg} / \mathrm{cm}^{-5}$, com pressão arterial sistólica $\geq$ a $80 \mathrm{mmHg}^{24}$. Classicamente esta estratégia requer ambiente de terapia intensiva com a utilização de cateter em artéria pulmonar. Redesenhando a terapia por 24 a $72 \mathrm{~h}$ de acordo com a hemodinâmica, utilizando essencialmente vasodilatador (nitroprussiato de sódio) e diurético de alça (furosemide) intravenosos, pode-se otimizar o perfil hemodinâmico e manter o paciente clinicamente estável por vários meses seguintes, com razoável melhora dos sintomas, permitindo, inclusive, sua retirada, mesmo que provisória, da lista de transplante cardía$\mathrm{co}^{25-28}$. Embora a estratégia de otimização hemodinâmica, utilizando cateter em artéria pulmonar tenha repetidamente trazido resultados com melhora de desfechos funcionais e clínicos, estes são dados observacionais, não testados em ensaio clínico. Neste sentido, o estudo multicêntrico, randomizado, denominado ESCAPE está em andamento, comparando a estratégia invasiva com manejo clínico na insuficiência cardíaca congestiva ${ }^{29}$. Estudos como esse devem responder se esta estratégia de fato altera desfechos, criando sobreviventes, ou simplesmente identifica aqueles com mais chances de sobreviver, independentemente, da estratégia terapêutica utilizada.

Além de proporcionar adequação do perfil hemodinâmico com esta abordagem, estudo recente demonstrou que nos pacientes com sério comprometimento hemodinâmico, intervenções com diuréticos e vasodilatadores, sem inotrópicos, dirigidas para normalizar as condições de sobrecarga e resistência sistêmica reduzem marcadores de ativação neurohumoral, como peptídeo natriurético atrial (ANP), peptídeo natriurético cerebral (BNP), e os vasoconstritores endotelina e norepinefrina ${ }^{30}$. Neste sentido, foi demonstrado que a intensificação da terapia da insuficiência cardíaca congestiva com diuréticos e inibidores da enzima de conversão da angiotensina (IECA) reduz os níveis séricos do BNP, e, além disso, pacientes tratados com terapia guiada por valores de BNP tiveram redução de eventos cardiovasculares de 35\% $(p=0,02)$ em relação ao grupo em acompanhamento clínico apenas ${ }^{31}$. Por outro lado, dados recentes indicam que 54\% dos pacientes com níveis basais de BNP de 480 pg/ml ou mais terão um novo episódio de congestão em 6 meses $^{32}$. Este conjunto de dados indica que guiar o tratamento da insuficiência cardíaca congestiva descompensa- 
da, objetivando reduções de pressões de enchimento, seja através de dados numéricos de hemodinâmica, seja através de redução de níveis de BNP (marcador que se eleva indicando distensão dos sarcômeros), traz benefícios clínicos para estes pacientes.

Ferramentas alternativas para caracterização de parâmetros hemodinâmicos - A imensa maioria dos dados preconizando a busca de otimização hemodinâmica advém de estudos utilizando cateter em artéria pulmonar para avaliação invasiva dos parâmetros hemodinâmicos. Evidentemente, que esta estratégia necessariamente se restringe a ambiente de terapia intensiva e se aplica àqueles pacientes em quadro de insuficiência cardíaca congestiva descompensada que buscaram atendimento hospitalar. Considerando a importância de manter os pacientes com insuficiência cardíaca congestiva euvolêmicos de forma a evitar ou protegê-los de descompensações por congestão, ferramentas para utilização em ambiente ambulatorial seriam desejáveis. Neste sentido, outros métodos não invasivos para avaliar débito cardíaco e pressões de enchimento, como ecocardiografia com Doppler e dispositivo de bioimpendância, podem ser instrumentos úteis para o acompanhamento e individualização da terapia da insuficiência cardíaca congestiva ${ }^{33,9}$.

Estudo recente utilizando um monitor hemodinâmico de pressões do ventrículo direito (Medtronic Incorporation) foi implantado como um marcapasso para guiar o manejo clínico diário em 32 pacientes com insuficiência cardíaca congestiva, e resultou numa redução de $57 \%$ das hospitalizações $(p<0,01)^{34}$.

Avaliação hemodinâmica ecocardiográfica - 0 ecocardiograma como ferramenta para avaliação hemodinâmica pode ser uma alternativa atraente, pois pode fornecer, na grande maioria dos pacientes em insuficiência cardíaca, dados hemodinâmicos com excelente correlação àqueles colhidos concomitantemente por cateterismo direito ${ }^{35,36}$. As pressões do lado direito, por exemplo, podem ser obtidas em $97 \%$ das vezes ao se avaliar as pressões diastólica (89\%) e sistólica (73,5\%) na artéria pulmonar ${ }^{37}$.

$\mathrm{Na}$ insuficiência cardíaca congestiva, estimativas de pressões de enchimento à direita podem ser feitas utilizando parâmetros ecocardiográficos, como por exemplo, o diâmetro da veia cava inferior associado ao seu índice de colapsabilidade na inspiração. A tendência da veia cava inferior de colapsar total ou parcialmente ocorre porque durante a inspiração aumenta a pressão negativa intratorácica, e desde que a veia cava inferior e as cavidades direitas não estejam sobrecarregadas, o fluxo de sangue deve aumentar em direção ao átrio direito, causando o colabamento parcial ou total desse vaso. Nos casos em que, por motivos técnicos, não for possível avaliar a veia cava inferior e não houver evidência de elevação de pressões em cavidades direitas, é consenso geral a utilização simplesmente de um valor arbitrário médio de $10 \mathrm{mmHg}^{38}$.

A pressão em artéria pulmonar pode ser obtida através da medida das velocidades das regurgitações entre cavidades direitas, basicamente regurgitação tricúspide e pulmonar. Com uma avaliação completa de todas as "janelas" ecocardiográficas é possível registrar o gradiente transvalvar trans-tricuspídeo e a regurgitação pulmonar em $86 \%$ e $89 \%$ dos exames, respectivamente. Foi demonstrado que adicionando as duas possibilidades, em $97 \%$ de uma série de 200 pacientes detectou-se algum valor pressórico em artéria pulmonar ${ }^{37,39}$. Em pacientes que não apresentam regurgitação pulmonar em grau suficiente para estimar gradiente, utiliza-se a regurgitação tricúspide no momento da abertura da válvula pulmonar como estimativa da pressão diastólica na artéria pulmonar. Esta técnica simples mostrou uma correlação de $r=0,92$ com o cateterismo cardíaco, excluindo-se os pacientes com insuficiência tricúspide severa e aqueles em ventilação mecânica ${ }^{40,41}$.

Outro importante dado hemodinâmico, o débito cardíaco, também pode ser calculado por dados ecocardiográficos, apresentando um coeficiente de correlação com os dados obtidos pelo cateterismo direito de $r=0,97^{35}$. Para tal, basta adquirir o produto da freqüência cardíaca pelo tempo da integral de velocidade (TVI) e pela área do trato da via de saída do ventrículo esquerdo, corrigindo pela superfície corporal para obter os valores de índice cardíaco.

A pressão capilar pulmonar é um parâmetro bem estabelecido na avaliação da função cardíaca e de enchimento ventricular esquerdo. Nos pacientes com insuficiência cardíaca congestiva, a pressão capilar pulmonar elevada está associada com um mau prognóstico, descompensações freqüentes dos sintomas e baixa tolerância ao exercício ${ }^{42-45}$. Redução destes valores após tratamento adequado melhora a qualidade de vida dos pacientes ${ }^{46}$. A utilização de dados referentes a estes valores pode auxiliar no manejo terapêutico rotineiro de pacientes com insuficiência cardíaca congestiva.

A pressão capilar pulmonar ou atrial esquerda podem ser estimadas pela avaliação da velocidade do fluxo na artéria pulmonar, caso o paciente não tenha doença pulmonar, ou pelo fluxo transmitral e venoso pulmonar do lado esquerdo. A análise do fluxo transmitral, também, fornece informações da pressão atrial esquerda e prognósticas na insuficiência cardíaca. No entanto, é dependente de múltiplas condições, como freqüência cardíaca, relaxamento e sucção ventricular, complacência atrial e ventricular esquerda e condições da válvula mitral. Para evitar a interferência destes fatores, outros parâmetros como o fluxo venoso pulmonar, a resposta do fluxo transmitral para diferentes cargas de volume, o modo $\mathrm{M}$ colorido, e o Doppler tecidual do ânulo mitral podem ser utilizados. A estimativa da pressão atrial esquerda também não pode ser feita com regras isoladas, mas sim após avaliação de vários parâmetros e respeitando as limitações de cada um. Para fins práticos considera-se que a pressão capilar pulmonar e a pressão de átrio esquerdo são equivalentes, porque os capilares pulmonares, as veias pulmonares e o átrio esquerdo se comunicam livremente, formando na verdade uma grande câmara. Medidas simultâneas de cateterismo direito e do átrio esquerdo através de cateterização transeptal já confirmaram a similaridade destas pressões ${ }^{47-49}$. Os diversos cálculos utilizados para obter dados hemodinâmicos através do ecocardiograma estão compilados na tabela I.

Outros métodos - O Doppler tecidual é uma técnica relativamente nova que registra as velocidades sistólica e diastólica do miocárdio ao nível do ânulo mitral, preferencialmente, medial ou lateral. Devem-se usar filtros para eliminar as altas freqüências e o limite de Nyquist deve ser mantido entre -15 a $20 \mathrm{~cm} / \mathrm{s}$. Ao contrário do fluxo transmitral (onda $\mathrm{E}$ ), a velocidade registrada no ânulo mitral pelo Doppler tecidual $\left(E_{a}\right)$ não sofre a influência da pressão atrial esquerda e sim reflete o relaxamento ventricular esquerdo isoladamente. Assim sendo, é um ótimo índice para avaliar déficit de relaxamento, mesmo nos casos pseudonormais, e quando acoplado ao fluxo transmitral não tecidual pode estimar a pressão atrial esquerda. Uma razão $E / E_{a}>10$ pode prever uma PAE > 12mmh com uma sensibilidade de $91 \%$ e uma especificidade de $81 \%$, ou uma relação $\mathrm{E}_{\mathrm{a}} \mathrm{E}_{\mathrm{a}}>10$ pode prever uma $\mathrm{PAE}$ $>15 \mathrm{mmhg}$ com uma sensibilidade de $97 \%$ e especificidade de 
Determinação de Parâmetros Hemodinâmicos Através do Ecocardiograma Bidimensional com Doppler: Ferramenta para Busca de Otimização Terapêutica em Pacientes Ambulatoriais com Insuficiência Cardíaca Congestiva

Tabela I - Fórmulas ecocardiográficas utilizadas para obtenção dos dados hemodinâmicos

\begin{tabular}{|c|c|}
\hline $\mathrm{DC}, \mathrm{L} / \mathrm{min}$ & VS x FC \\
\hline $\mathrm{VS}, \mathrm{mL}$ & TVI x AVSVE \\
\hline AVSVE, $\mathrm{cm}^{2}$ & $0,785 D^{2}$ \\
\hline íc, L/min/m² & $\mathrm{DC} / \mathrm{SC}$ \\
\hline $\mathrm{SC}, \mathrm{m}^{2}$ & [altura $(\mathrm{cm})+$ peso $(\mathrm{kg})]-60 / 100$ \\
\hline ÍRVS, dinas.cm ${ }^{-5}$ & $\mathrm{PAM}-\mathrm{PAD} / \mathrm{IC} \times 80$ \\
\hline PAE, mmHg (Henry) & $65-(0,5 \mathrm{TAP})$ \\
\hline PAE, mmHg (Dabestani) & $57-(0,39$ TAP $)$ \\
\hline PAE, mmHg (TRIV e Vp) & $4,5\left(10^{3} /[2\right.$ TRIV] $+V p)-9$ \\
\hline $\begin{array}{l}\mathrm{PAE}, \mathrm{mmHg} \text { (Vp e velocidade } \\
\text { máxima onda } \mathrm{E} \text { ) }\end{array}$ & $5,27[E / V p]+4,6$ \\
\hline PAE, mmHg (Doppler tecidual) & $1,24\left(E / E^{\prime}\right)+1,9$ \\
\hline PSAP, mmHg & $\triangle A D / V D+P A D$ \\
\hline PDAP, mmHg & $\triangle \mathrm{PDF} I \mathrm{P}+\mathrm{PAD}$ \\
\hline PMAP, $\mathrm{mmHg}$ & $90-(0,6 \mathrm{TAP})$ \\
\hline $\mathrm{PAD}, \mathrm{mmHg}$ & $\varnothing \mathrm{VCl}+\%$ colapso inspiratório \\
\hline
\end{tabular}

DC - débito cardíaco; VS - volume sistólico; TVI - integral da velocidade do fluxo; AVSVE - área na via de saída do ventrículo esquerdo (VE); D diâmetro na via de saída do VE; IC - índice cardíaco; SC - superfície corporal; IRVS - índice da resistência vascular sistêmica; PAM - pressão arterial média; PAD - pressão estimada no átrio direito; $P A E$ - pressão estimada no átrio esquerdo; TAP - tempo de aceleração do fluxo em artéria pulmonar; TRIV - tempo de relaxamento isovolumétrico; Vp velocidade de propagação do fluxo transmitral pelo modo color $\mathrm{M} ; \mathrm{E}$ velocidade máxima do fluxo transvalvar mitral; E' - velocidade máxima da onda E tecidual; PSAP - pressão sistólica na artéria pulmonar; $\triangle A D$ / VD - gradiente de pressão na regurgitação tricúspide; PDAP - pressão diastólica na artéria pulmonar; PMAP - pressão média na artéria pulmonar; $\triangle$ PDF IP - gradiente de pressão diastólico final na insuficiência pulmonar; $\varnothing \mathrm{VCl}$ - diâmetro da veia cava inferior.

78\%. Ommen e cols. ${ }^{50}$ demonstraram um nível de correlação para a relação $E / E_{a \text { medial }}$ e a pressão média diastólica do ventrículo esquerdo de $r=0,64$, maior nos pacientes com fração de ejeção menor do que 50\%, comparativamente, àqueles sem disfunção ventricular. Esses autores também demonstraram um valor preditivo positivo de $64 \%$ para uma pressão diastólica final do ventrículo esquerdo $>$ de $12 \mathrm{mmHg}$ se $\mathrm{E}_{\mathrm{a}} \mathrm{E}_{\mathrm{a}}>15 \mathrm{mmHg}$ e um valor preditivo negativo de $97 \%$ para pressões baixas no átrio esquerdo se a relação $E / E_{a}$ for < 8. Na presença de infarto da parede lateral basal, fibrilação atrial e insuficiência mitral severa há limitações técnicas importantes que podem inviabilizar estas medidas ${ }^{51,52}$.

Além dos métodos citados, várias outras fórmulas podem ser utilizadas na estimativa da pressão atrial esquerda, utilizando os seguintes parâmetros ecocardiográficos: tempo de relaxamento isovolumétrico, velocidade de propagação do fluxo transmitral obtida pelo color modo M (VFTM) ${ }^{53-57}$, velocidade de desaceleração do fluxo transvalvar mitral precoce ${ }^{58-60}$, fração sistólica do fluxo venoso pulmonar e tempo de desaceleração do fluxo pulmonar diastólico ${ }^{61}$, fração sistólica da integral de velocidade do fluxo anterógrado venoso pulmonar ${ }^{62}$ e relação entre o tempo de contração atrial do fluxo retrógrado venoso pulmonar e o anterógrado transmitral ${ }^{63}$. A escolha da técnica mais apropriada pode variar conforme características individuais dos pacientes.

Portanto, a avaliação hemodinâmica não invasiva, com as várias informações fornecidas pela ecocardiografia, pode ser obtida com boa acurácia utilizando-se mais de um parâmetro e conhecendo-se as vantagens e limitações de cada um dos métodos. Tecnicamente requer tempo e treinamento do ecocardiografista, e sua realização está ligada à necessidade imposta pelo cardiolo-
Aplicação clínica do uso do ecocardiograma para buscar parâmetros hemodinâmicos otimizados - 0 nosso grupo está conduzindo um ensaio clínico randomizado comparando tratamento ambulatorial guiado por ecocardiografia, objetivando adequação do perfil hemodinâmico (redução de pressões de enchimento e da resistência periférica) versus manejo clínico convencional objetivando melhora sintomática, em que foram arrolados 99 pacientes com diagnóstico de insuficiência cardíaca congestiva de qualquer etiologia, em classe funcional II-IV, com fração de ejeção $\leq a$ $40 \%$ que tiveram internação hospitalar ou visita a emergência por insuficiência cardíaca descompensada nos últimos três meses. Dados preliminares de 70 pacientes com idade média de 60 \pm 15 anos, $60 \%$ do sexo masculino, fração de ejeção de $27 \pm 7 \%$ e $31 \%$ de etiologia isquêmica, demonstraram que no grupo de tratamento guiado por ecocardiografia, houve redução da pressão atrial direita de $10,1 \pm 5$ para $7,8 \pm 4$ mmh $(p=0,004)$, da pressão sistólica máxima da artéria pulmonar de $47 \pm 12$ para $39 \pm 12$ mmhg $(p=0,003)$ e do índice da resistência vascular sistêmica de $3821 \pm 1265$ para $3390 \pm 1142$ dinas $/ \mathrm{s} / \mathrm{cm}^{-5}(p=0,048)$. Não houve diferença significativa entre esses parâmetros no grupo com manejo clínico convencional. Estes dados e a condução deste estudo até o momento indicam que o emprego da ecocardiografia para guiar tratamento baseado em dados hemodinâmicos é factível e mais eficaz do que o tratamento baseado em manejo clínico tradicional para obtenção de um perfil favorável de redução de pressões de enchimento e da resistência periférica em pacientes ambulatoriais com insuficiência cardíaca.

Papel do perfil hemodinâmico no prognóstico na insuficiência cardíaca crônica e fatores preditivos - A importância do estado volêmico no prognóstico dos pacientes com insuficiência cardíaca congestiva pode ser também observada nos dados que indicam que sobrevida destes pacientes varia entre $80 \%$ em dois anos para aqueles livres de congestão até menos do que 50\% em 6 meses nos com sintomas refratários em repouso ${ }^{64}$. A manutenção de um estado clínico livre de congestão, buscando níveis de pressões de enchimento baixas ou normais dentro de 4 a 6 semanas após a alta hospitalar de pacientes em classe funcional IV se associou com melhor sobrevida nos 2 anos seguintes ${ }^{65}$.

A monitorização hemodinâmica também pode ser útil para estratificar risco, pois pacientes que apresentavam pressões de enchimento iniciais normais tiveram uma sobrevida em um ano de $95 \%$, enquanto nos com pressões iniciais elevadas, foi sucesso a terapia em reduzir as pressões que se correlacionaram com melhor prognóstico e não o nível de congestão inicial ${ }^{66}$.

Além do estado congestivo dos pacientes, outros informações prognósticas importantes na insuficiência cardíaca congestiva podem ser obtidas por ecocardiograma, incluindo: grau de preservação da função ventricular direita ${ }^{67,68}$, diâmetro diastólico final do ventrículo esquerdo ${ }^{69}$, pressão atrial esquerda, padrão restritivo no fluxo diastólico transmitral ${ }^{70-72}$ e graus de insuficiência mitral e tricúspide ${ }^{73,74}$. Níveis de BNP, que estão elevados na insuficiência cardíaca congestiva talvez indicando grau de congestão, também se correlacionam com morte súbita ${ }^{75}$, classe funcional da $\mathrm{NYHA}^{76}$ e eventos futuros ${ }^{77}$.

Pressões de enchimento, portanto, são úteis no manejo dos pacientes em insuficiência cardíaca congestiva porque podem predizer desfechos mesmo após ajustes da terapia ${ }^{66}$ e podem ser inferidas não só pelos níveis de BNP, mas também através de parâmetros ecocardiográficos, como os já citados anteriormente. 
Considerações finais - 0 tratamento da disfunção cardíaca sistólica tem avançado consideravelmente nos últimos 20 anos, devido, principalmente, às descobertas no campo patofisiológico do papel dos fatores neurohormonais ${ }^{78}$; levando à utilização de drogas comprovadamente redutoras de mortalidade como IECA ${ }^{79-81}$, beta-bloqueadores $^{82,83}$ e espironolactona ${ }^{84}$. A atuação de equipes multidisciplinares tem sido importante para especificamente atuar na redução das readmissões hospitalares ${ }^{85,86}$.

Apesar destes avanços, ainda são altas a mortalidade e os níveis de readmissão hospitalar por descompensação dos sintomas $^{87,88}$, o que se deve, parcialmente, à limitação do quadro clínico para indicar adequação do tratamento pré-alta, especialmente, na definição do estado congestivo/volêmico desses pacientes. Testes convencionais, como a ecocardiografia, não são utilizados de rotina na sua potencialidade plena para fornecer informações adicionais ao exame clínico, em relação às reais condições hemodinâmicas do paciente. Por outro lado, a utilização frequente de cateterização cardíaca direita no manejo de pacientes com insuficiência cardíaca descompensada é inexeqüível, não só pelos riscos e altos custos do procedimento, mas também porque esta implica em utilização de leitos em terapia intensiva ${ }^{89-92}$. Neste sentido, métodos alternativos devem ser buscados para trazer informações pertinentes ao estado volêmico dos pacientes com insuficiência cardíaca congestiva. A dosagem do neurohormônio BNP tem mostrado resultados iniciais positivos para monitorar o tratamento ambulatorial em longo prazo destes pacientes. A ampla disponibildade do ecocardiograma atualmente na clínica cardiológica poderia permitir um melhor controle ambulatorial de pacientes com insuficiência cardíaca congestiva, fosse o potencial deste método para determinar perfil hemodinâmico dominado de forma mais abrangente por cardiologistas/ecocardiografistas, que poderiam fornecer informações preciosas ao clínico permitindo um padrão de manejo diferenciado para este difícil grupo de pacientes.

\section{Referências}

1. Roglieri JL, Futterman R, McDonough KL et al. Disease management intervention to improve outcomes in congestive heart failure. Am J Mang Care 1997;3:1831-9.

2. McAlister FA, Lawson $\mathrm{FM}$, Teo $\mathrm{KK}$ et al. A systematic review of randomized trials of disease management programs in heart failure. Am J Med 2001;110:378-84.

3. Hunt AS, Baker DW, Chin MH, Cinquegrani MP et al. ADD/AHA Guidelines for the evaluation and management of chronic heart failure in the adult. J Am Coll Cardiol 2001;38:2101-13.

4. Drazner MH, Rame JE, Stevenson, LW, Dries DL. Prognostic importance of elevated jugular venous pressure and a third heart sound in patients with heart failure. The New Engl J Med 2001; 345, 574-81.

5. Shah NB, Der E, Ruggerio C, Heindenreich PA et al. Prevention of hospitalizations for heart failure with an interactive home monitoring program. Am Heart J 1998; 135:373-8

6. Stevenson LW, Perloff J. The limited reliability of physical signs for estimating hemodynamics in chronic heart failure. JAMA 1989;261:884-88.

7. McGee SR. Physical examination of venous pressure: a critical review. Am Heart J 1998;136:10-18.

8. Drazner MH, Hamilton MA, Fonarow $\mathrm{G}$ et al. Relationship between right and left -sided filling pressures in 1000 patients with advanced heart failure. J Heart Lung Transplant 1999;18:1126-32.

9. Badgett RG, Lucey CR, Mulrow CD. Can the clinical examination diagnose left-sided heart failure in adults? JAMA 1997;277:1712-9.

10. Butman SM, Ewy GA, Standen JR et al. Bedside cardiovascular examination in patients with severe chronic heart failure: importance of rest or inducible jugular venous distension. J Am Coll Cardiol 1993;22:968-74.

11. Zema MJ, Restivo B, Sos T et al. Left ventricular dysfunction: bedside Valsalva maneuver. Br Hear J 1980;44:560-9.

12. Westman EC, Matchar DB, Samsa GP, Mulrow CD et al. Accuracy and reliability of apical S3 gallop detection. J Gen Intern Med 1995;10:455-7.

13. Lok GE, Morgan $C D$, Ranganathan $N$. The accuracy and interobserver agreement in detecting the "gallop sounds" by cardiac auscultation. Chest 1998; 114: 1283-8.

14. Azevedo ER, Newton GE, Floras JS et al. Reducing cardiac filling pressure lowers norepinephrine spillover in patients with chronic heart failure. Circulation 2000; 101:2052-9.

15. Kaye DM, Lambert GW, Lefkovits J, Morris M, Jennings G, Esler MD. Neurochemical evidence of cardiac sympathetic activation and increased central nervous system norepinephrine turnover in severe congestive heart failure. J Am Coll Cardiol 1994; 23:570-8.

16. Weilenmann D, Hans Rickli, Follath Ferenc et al. Noinvasive evaluation of pulmonary capillary wedge pressure by BP response to the Valsalva maneuver. Chest $2002 ; 122,140-5$

17. Rosario LB, Stevenson LW, Solomon SD, Lee RT, Reimold SC. The mechanism of decrease in dynamic mitral regurgitation during heart failure treatment: importance of reduction in the regurgitant orifice size. J Am Coll Cardiol 1998; 32:1819-24.

18. Rosario LB, Stevenson LW, Chelimsky-Fallick C et al. Sustained hemodynamic efficacy of therapy tailored to reduce filling pressures in survivors with advanced heart failure. Circulation 1997; 96:1165-72.

19. Johson W, Omland T, Collins CM et al. Neurohormonal activation rapidly decreases after intravenous vasodilator and diuretic therapy for class IV heart failure [abstract]. Circulation 1998; 98:I-780.
20. Lucas C, Johnson W, Hamilton MA et al. Freedom from congestion predicts good survival despite previous class IV symptoms of heart failure. Am Heart J 2000; 140:840-7.

21. Chomsky DB, Lang CC, Rayos G et al. Treatment of subclinical fluid retention in patients with symptomatic heart failure: effect of exercise performance. J Heart Lung Transplant 1997; 16:846-53.

22. Carr JG, Stevenson LW, Walden JÁ et al. Prevalence and hemodynamic correlates of malnutrition in severe congestive heart failure secondary to ischemic or idiopathic dilated cardiomyopathy. Am J Cardiol 1989;63:709-13.

23. Stevenson LW, Bellil D, Grover-Mckay et al. Effects of afterload reduction on left ventricular volume and mitral regurgitation in severe congestive heart failure secondary to ischemic or idiopathic dilated cardiomyopathy. Am J Cardiol 1987; 60: 654-8.

24. Stevenson $\mathrm{LW}$, Tillisch JH. Maintenance of cardiac output with normal filling pressures in patients with dilated heart failure. Circulation 1986; 74, 1303-8.

25. Pierpont GL, Cohn J, Franciosa JA. Combined oral hydralazine-nitrate therapy in left ventricular failure: hemodynamic equivalency to sodium nitroprusside. Chest 1978;73:8-13

26. Rohde LE, Furian T, Campos C et al. Implications of the hemodynamic optimization approach guided by right heart catheterization in patients with severe heart failure. Arq Bras Cardiol 2002;78:261-6.

27. Stevenson $\mathrm{L}$. Tailored therapy to hemodynamic goals for advanced heart failure. European J of Heart Failure 1999; 1: 251-7.

28. Steimle AE, Stevenson LW, Chelimsky-Fallick C et al. Sustained hemodynamic efficacy of therapy tailored to reduce filling pressures in survivors with advanced heart failure. Circulation 1997;96, 1165-71.

29. Shah MR, O'Connor CM, Sopko G et al. Evaluation study of congestive heart failure and pulmonary artery catheterization effectiveness (ESCAPE): design and rationale. Am Heart J 2001;141:528-35.

30. Johnson W, Ormland T, Hall C et al. Neurohormonal activation rapidly decreases after intravenous therapy with diuretics and vasodilators for class IV heart failure. J Am Coll Cardiol 2002; 39:1623-9.

31. Troughton RW, Frampton CM, Yandle TG, Espiner EA et al. Treatment of heart failure guided by plasma aminoterminal brain natriuretic (N-BNP) concentrations. Lancet 2000; 355:1126-30.

32. Maisel, AS, Krishnaswamy P, Nowak RM et al. Rapid measurement of B-type natriuretic peptide in the emergency diagnosis of heart failure. N Engl J Med 2002; 347:161-7.

33. Cheng V, Kazanagra R, Garcia A et al. A rapid bedside test for B-type peptide predicts treatment 2001;37:386-91.

34. Adamson PB, Magalski A, Braunschweig F et al. Ongoing right ventricular hemodynamics in heart failure. J Am Coll Cardiol 2003;41:565-71.

35. Stein JH, Neumann A, Preston LM, Costanzo MR et al. Echocardiography for hemodynamic assessment of patients with advanced heart failure and potential heart transplant recipients. J Am Coll Cardiol 1997; 30:1765-72.

36. Dini FL, Bezante GP, Faggiano P, Odaglia F et al. Is a totally non-invasive assessment of the hemodynamic profile possible in patients with chronic heart failure? Ital Heart J 2000; 1:1395-403.

37. Borgeson DD, Seward JB, Miller FA, Oh JK, Tajik J. Frequency of Doppler measurable pulmonary artery pressures. J Am Soc Echocardiogr 1996;9:832-7.

38. Moreno FL, Hagan AD, Holmen JR, Pryor TA, Strickland RD, Castle CH. Evaluation 
Determinação de Parâmetros Hemodinâmicos Através do Ecocardiograma Bidimensional com Doppler: Ferramenta para Busca de Otimização Terapêutica em

Pacientes Ambulatoriais com Insuficiência Cardíaca Congestiva

of size and dynamics of the inferior vena cava as an index of right-sided cardiac function. Am J Cardiol 1984;53:579-85.

39. Abramson SV, Burke JB, Pauletto FJ, Kelly JK. Use of multiple views in the echocardiographic assessment of pulmonary artery systolic pressure. J Am Soc Echocardiogr 1995;8:55-60.

40. Stephen B, Dalal P, Berger M, Schweitaer P, Hecht S. Noninvasive estimation of pulmonary artery diastolic pressure in patients with tricuspid regurgitation by Doppler echocardiography. Chest 1999; 116: 73-77.

41. Steven JL. Noninvasive estimation of right-sided pressures from spectral Doppler recordings of tricuspid and plutonic regurgitation velocities. Editorial: chest 1999; 116: 1-3.

42. Werner GS, Schaefer C, Dirks R et al. Prognostic value of Doppler echocardiographic assessment of left ventricular filling in idiopathic dilated cardiomyopathy. Am J Cardiol 1994;73:792-8.

43. Franciosa JÁ, Backer BJ, Seth L. Pulmonary versus systemic hemodynamics in determining exercise capacity of patients with chronic left ventricular failure. Am Heart J 1985;110:807-13.

44. Vanoverscherlde JLJ, Raphael DA, Robert AR, Cosyns R. Left ventricular filling in dilated cardiomyopathy: relation to functional class and hemodynamics. J Am Coll Cardiol 1990;15:1288-95.

45. Stevenson LW, Tillish JH. Maintenance of cardiac output with normal filling pressures in patients with dilated heart failure. Circulation 1986;74:1303-8.

46. Stevenson LW. Tailored therapy before transplantation for treatment of advanced heart failure: effective use of vasodilators and diuretics. J Heart Lung Transplant 1991;10:468-76.

47. Walstson A, Kendall ME. Comparison of pulmonary wedge and left atrial pressure in man. Am Heart J 1973;86:159-64.

48. Luchsinger PC, Seipp HW, Patel DJ. Relationship of pulmonary artery-wedge pressure to left atrial pressure in man. Circ Res 1962; 11:315-8.

49. Braunwald E, Drockenbrough ED, Frahm CJ, Ross J. Left atrial and left ventricular pressures in subjects without cardiovascular disease. Circulation 1961; 24: 267-9.

50. Ommen SR, Nishimura RA, Appleton CP, Miller FZ, Oh JK, Redfield MM, Tajik AJ. Clinical utility of Doppler echocardiography and tissue Doppler imaging in the estimation of left ventricular filling pressures. Circulation 2000;102:1788-94.

51. Nagheh SF, Middleton KJ, Kopelen HÁ, Zoghbi WA, Quiñones MA. Doppler tissue imaging: a noninvasive technique for evaluation of left ventricular relaxation and estimation of filling pressures. J Am Coll Cardiol 1997;30:1527-33.

52. Gorcsan III J. Tissue Doppler echocardiography. Lippincott Williams \& Wilkins, Inc. Cur Opin Cardiol 2000,15:323-9.

53. Brun P, Tribouilloy $C$, Duval AM et al. Left ventricular flow propagation during early filling is related to wall relaxation: a color M-mode Doppler analysis. J Am Coll Cardiol 1992;20:420-32.

54. Takasuji H, Mikami T, Urasawa K et al. A new approach for evaluation of left ventricular diastolic function: spatial and temporal analysis of left ventricular filling flow propagation by color M-mode Doppler echocardiography. J Am Coll Cardiol 1996;27:365-71.

55. Duval-Moulin AM, Dupouy P, Brun P et al. Alteration of left ventricular diastolic function during coronary angioplasty-induced ischemia: a color M-mode Doppler study. J Am Coll Cardiol 1997;29:1246-55.

56. Weiss JL, Ares MA, Asher $\mathrm{C}$ et al. An index of early left ventricular filling that combined with pulsed Doppler peak E velocity may estimate capillary wedge pressure. J Am Coll Cardiol 1997;29:448-54.

57. Gonçales- Vilchez F, Ares M, Ayuela J, Alonso L. Combined use of pulsed and color M-mode Doppler echocardiography for the estimation of pulmonary capillary wedge pressure: an empirical approach based on an analytical relation. J Am Coll Cardiol 1999;34:515-23.

58. Garcia MJ, Ares MA, Asher C et al. An index of Early left ventricular filling that combined with pulsed Doppler peak E velocity may estimate capillary wedge pressure. J Am Coll Cardiol 1997;29: 448-54.

59. Pozzoli M, Capomolla S, Pinna G, Cobelli F, Tavazzi L. Doppler echocardiography reliably predicts pulmonary artery wedge pressure in patients with chronic heart failure with and without mitral regurgitation. J Am Coll Cardiol 1996; 27:883-93.

60. Gianuzzi P, Imparato A, Temporelli PL et al. Doppler- derived mitral desaceleration time of early filling as a strong predictor of pulmonary capillary wedge pressure in post-infarction patients with left ventricular systolic dysfunction. J Am Coll Cardiol 1994;23:1630-7.

61. Yamamuro A, Yoshida K, Hozumi T et al. Noninvasive evaluation of pulmonary capillary wedge pressure in patients with acute myocardial infarction by deceleration time of pulmonary venous flow velocity in diastole. J Am Coll Cardiol 1999; 34: 90-4.

62. Brunazzi MC, Chirillo F, Pasqualini M et al. Estimation of left ventricular diastolic pressures from precordial pulsed-Doppler analysis of pulmonary venous and mitral flow. Am Heart J 1994;128:293-300.

63. Rossvoll O, Hatle LK. Pulmonary venous flow velocities recorded by transthoracic Doppler ultrasound: relation to left ventricular diastolic pressures. J Am Coll Cardiol 1993;21:1687-96.

64. Uretsky BF, Sheahan RG. Primary prevention of sudden cardiac death in heart failure: will the solution be shocking? J Am Coll Cardiol 1997; 30:1589-97.
65. Caroline L, Johnson W, Hamilton MA et al. Freedom from congestion predicts good survival despite previous class IV symptoms of heart failure. Am Heart J 2000; 140:840-7.

66. Stevenson LW, Tillisch JH, Hamilton M et al. Importance of hemodynamic response to therapy in predicting survival with ejection fraction $<20 \%$ secondary to ischemic or nonischemic dilated cardiomyopathy. Am J Cardiol 1990; 66:1348-54.

67. Di Salvo TG, Mathier M, Semigran MJ, Dec GW. Preserved right ventricular ejection fraction predicts exercise capacity and survival in advanced heart failure. J Am Coll Cardiol 1995; 25: 1143-53.

68. Lewis JF, Webber JD, Sutton LL, et al: Discordance in degree of right and left ventricular dilatation in patients with dilated cardiomyopathy: Recognition and clinical implications. J Am Coll Cardiol 1993;21:649-54.

69. Gavazzi A, DeMaria R, Renosto $G$ et al. The spectrum of left ventricular size in dilated cardiomiopathy: clinical correlates and prognostic implications. Am Heart J 1993;125:410-22.

70. Xie GY, Martin RB, Smith MD et al. Prognostic value of Doppler transmitral flow patterns in patients with congestive heart failure. J Am Coll Cardiol 1994; 24:132-9.

71. Pinamonti B, DiLenarda A, Sinagra G, Camerii F. Restrictive left ventricular filling pattern in dilated cardiomyopathy assessed by Doppler echocardiography: clinical, echocardiography and hemodynamic correlations and prognostic implications. Heart Muscle Disease Study Group. J Am Coll Cardiol 1993; 22:808-15.

72. Lapu-Bula R, Robert A, De Kock M et al. Risk stratification in patients with dilated cardiomyopathy: Contribution of Doppler-derived left ventricular filling. Am J Cardiol 1998;82:779-85.

73. Abrahmson SV, Burke JF, Kelly JJ et al. Pulmonary hypertension predicts mortality and morbidity in patients with dilated cardiomyopathy. Ann Intern Med 1992; 116:888-95.

74. Hung J, Koellin T, Semigran MJ et al. Usefulness of echocardiographic determined tricuspid regurgitation in predicting event free survival in severe heart failure secondary to idiopathic-dilated cardiomyopathy or to ischemic cardiomyopathy. Am J Cardiol 1998;82:1301-3.

75. Berger R, HuesIman M, Strecker K et al. B-type natriuretic predicts sudden death in patients with chronic heart failure. Circulation 2002;105:2392-7.

76. Clerico $A$, Lervasi $G$, Chicca $M$ et al. Circulating levels of cardiac natriuretic peptides (ANP e BNP) measured by highly sensitive and specific immunoradiometric assays in normal subjects and in patients with different degrees of heart failure. J Endocrine Invest 1998; 21:170-9.

77. Cheng V, Kazanagra R, Garcia A et al. A rapid bedside test for B-type peptide predicts treatment outcomes in patients admitted for decompensated heart failure: a pilot study. J Am Coll Cardiol 2001;37:386-91.

78. Remme WJ. Therapeutic strategies and neurohormonal control in heart failure. Eur Heart F 1994;15 (suppl D): 129-38.

79. The SOLVD Investigators. The effect of enalapril on survival in patients with reduced left ventricular ejection fraction and congestive heart failure. N Engl J Med 1991;325:293-302.

80. Garg R, Yusuf S. Overview of randomized trials of angiotensin-converting enzyme inhibitors on morbidity and mortality in patients with heart failure: Collaborative Group on ACE-Inhibitor Trials. JAMA 1995;273:1450-6.

81. The CONSENSUS Trial Study Group. Effects of enalapril on mortality in severe congestive heart failure: results of the Cooperative North Scandinavian Enalapril Survival Study (CONSENSUS). N Engl J Med 1987;316:1429-35.

82. CIBIS II Investigators Committees. The Cardiac Insufficiency Bisoprolol Study II (CIBIS II): a randomized trial. Lancet 1999;353:9-13.

83. MERIT-HF Study Group. Effect of metoprolol CR/XL. Randomised Intervention Trial in Congestive Heart Failure (MERIT-HF). Lancet 1999;353:2001-1007.

84. Pitt B, Zannad F, Remme WJ et al. The effect of spironolactona on morbidity and mortality in patients with severe heart failure. N Engl J Med 1999; 341: 709-717.

85. Kornowsky R, Zeeli D, Averbuch M et al. Intensive home-care surveillance prevents hospitalization and improves morbidity rates among elderly patients with severe congestive heart failure. Am Heart J 1995; 129:762-66.

86. Rich MW, Beckham V, Wittnberg C et al. A multidisciplinary intervention to prevent the readmission of elderly patients with congestive heart failure. N Engl J Med 1995;333:1190-95.

87. Philbin EF, DiSalvo TG. Prediction of hospital readmission for heart failure: Development of a simple risk score based on administrative data. J Am Coll Cardiol 1999;33:1560-6.

88. Drumholtz HM, Chen YT, Wan Y et al. Predictors of readmission among elderly survivors of admission with heart failure. Am Heart J 2000;139:72-7.

89. Adams KF: Post hoc subgroup analysis and the truth of a clinical trial. Am Heart J 1998;136:751-8

90. Stevenson LW, Massie BM, Francis GS. Optimizing therapy for complex or refractory heart failure: a management algorithm. Am Heart J 1998; 135(suppl): S293-309.

91. Stevenson LW: Therapy tailored for symptomatic heart failure. Heart Failure 1995; 22:955-62

92. Connors AF Jr, Speroff T, Dawson NV, et al. The effectiveness of right heart catheterization in the initial care of critically ill patients. JAMA 1996;276:889-97. 